\title{
COMPLEXITY AND INTRANSITIVITY IN TECHNOLOGICAL DEVELOPMENT
}

\author{
Alexander Y. KLIMENKO \\ The University of Queensland, SoMME, Qld 4072, Australia \\ klimenko@mech.uq.edu.au
}

\begin{abstract}
This paper investigates the existence of a fundamental link between two disciplines that emerged during last few decades: complexity science and advanced engineering. During this time many industries, especially those related to the high-tech end of technological development, have faced the problem of increasing complexity of design, production and operation. Industrial projects have grown to become multidisciplinary, tightly interconnected, costly and difficult to control and predict. Two trends can be identified in this respect: one is the consistent effort of systems engineering in reducing the uncertainties of complex industrial operations and the other is the effort undertaken in complexity studies to account for uncertainties present in the real world.

In this work, we provide a brief overview of recent developments in advanced engineering and give a consistent interpretation of technological evolution from the perspective of complexity science in general and complex competitive systems (CCS) in particular. CCS is a general framework that was recently developed for analysis of complex systems involving competition. Transitivity of the decision-making process and the cyclic nature of technological progress are considered. Correctness of intransitive decisions is inherently relativistic: the same decisions can be seen as correct or incorrect when considered from different perspectives. When treated simplistically, intransitivity may seem to be illogical but, nevertheless, it is common in nature and needs to be studied. CCS provides a formalised scientific framework for analysis of intransitivity and establishes the existence of an important connection linking complexity and uncertainty with intransitivity. Implications of intransitivity for engineering decision-making and strategic planning are considered in the context of CCS. A working example of intransitivity in competition between major car manufacturers is presented.
\end{abstract}

Keywords: Complexity, Technology strategy, Systems Engineering, Industrial strategy and decisionmaking, Complex competitive systems.

\section{Introduction}

The $20^{\text {th }}$ century was the century of specialisation. Radical advances in science, which marked the beginning of that century, developed into accomplished scientific fields requiring specialised expert knowledge. Communications between different disciplines became rare giving way to highly specialised terminology and approaches, while scientific disciplines have been fragmented into isolated fields. The two major discoveries of $20^{\text {th }}$ century physics - general relativity and quantum mechanics - do not form a unified and consistent picture of the universe. However, new forces - the forces of integration --- became visible towards the end of the century: the very large (cosmology) appears to be connected to the very small (elementary particle physics), economists are now working with mathematicians, and the new science of complexity seems to cross all possible borders of the traditional disciplines. 
Similar trends are observed in engineering. Rapid development of technology in the last century has segmented the engineering discipline into numerous fields: mechanical, electrical, mining, chemical, aerospace, nuclear and many others but the last decades of the $20^{\text {th }}$ century brought clear integration trends, which stimulated cross-disciplinary engineering research and links. Large corporations, which commonly have to run technologically diverse and very complex engineering projects, face a need for employing many specialists representing different fields of knowledge as well as the necessity to integrate numerous parts and subsystems into a functioning, reliable and efficient final product (Berkun, 2007). The latter task is entrusted to systems engineering (SE), which was born in response to the integration trends and complexity of modern technological development (Hall, 1962).

In analysis of these changes, we might observe two opposite trends: SE evolved towards its more practical, narrowly defined and predictable version (NASA, 1995; INCOSE, 2013), while uncertainty became a commonly recognised factor in studies of complex systems (Holland, 2006; Heylighen et al, 1999). This work follows the connection of advanced engineering to science of complex systems (Hall, 1962, Heylighen et al, 1999), while focusing on technological-type cycles (Kondratiev, 1925; Perez, 2010) and its connection to the phenomenon of intransitivity (Condorcet, 1785; Arrow, 1951). We use the term "advanced engineering" to distinguish the original broad interpretation of SE (Hall, 1962) grouped with associated fields of science and engineering from more narrowly defined modern and practical version of this discipline (NASA, 1995). The perspective offered by abstract competition and complex competitive systems (CCS - see Klimenko, 2013) is deployed in our considerations.

The rest of the paper is organised as follows. In Section 2 of the present work we review the existing links between complexity and advanced engineering. Patterns of technological development are considered in Section 3 from the perspective of CCS, which links complexity observed in competitive systems to intransitivity of the competition rules. Section 4 introduces a working example from automotive industry and discusses further intransitive aspects of decision-making. Conclusions are presented in Section 5.

\section{Technological Complexity}

\subsection{Complex Systems}

Despite the existence of quite obvious common sense interpretations of this term, an optimal definition of complex systems is yet to be established. Variations in the understanding of complexity and different definitions of complex systems have been repeatedly discussed in the literature (see relevant reviews in Heylighen et al, 1999).

We commonly use the word "system" to denote something which consists of semiautonomous parts, i.e. distinguishable elements that work in interaction with each other and with the environment. This definition is interdisciplinary and does not put any restrictions on the physical nature of the system under consideration. Hence, a bicycle is a mechanical system, a power grid is an electrical system and a human being is a biological system. System elements are often also represented by systems, whose elements might be systems as well (i.e. system can be a "system of systems") Hierarchies of systems of many different levels are common for complex systems.

A complex system is a system that has emergent properties, that is properties that cannot be easily predicted or derived from workings of the system elements. A bicycle is not a complex system as its functioning is fully predictable, a power grid is likely to have some complex features, and a human being is truly complex as it is impossible to predict human behaviour from the workings of biological parts. Thus a complex system

- consists of a large number of elements

- possesses emergent properties and

- is fully or partially unknown

The term "unknown" is present in the definition to stress the common understanding of complexity as something difficult to predict. French (2013) has connected this understanding of complexity to the Rumsfeld classification of unknowns ${ }^{1}$. Consider the

\footnotetext{
${ }^{1}$ Here we refer to the unexpected contribution to the philosophy of science from the former US defence secretary Donald Rumsfeld, who has profoundly
} 
following example: gas in a cylinder is comprised of many elements - molecules and has an emergent property, which is described by the second law of thermodynamics. This property can not be inferred from collisions of few molecules, which remain perfectly reversible in time. A large number of molecules possesses the property of increasing entropy, which, although not in contradiction with mechanical laws controlling behaviour of the distinct molecules, can not be derived from these laws alone. Should we call this system complex? Generally, yes we should: interactions of molecules are indeed very complex when considered from a mechanical perspective. We, however, happen to know well the main outcome of these interactions - the state of thermodynamic equilibrium, represented by canonical distributions. This knowledge makes us think that gas in a cylinder is not that complex.

Here we encounter a general feature of human knowledge: things that we do not understand seem complex to us but, once an adequate explanation is found, we see these things as being not so complex after all. Canonical (Gibbs) distributions represent a system property, which in addition to conventional thermodynamics can be encountered in different physical circumstances: two-dimensional turbulence, probability measures on graphs, and competitive systems (see Klimenko, 2012). We understand these distributions quite well and do not see them as complex.

The definition of complex systems given above suffers from being subjective due to the last item in the definition - complexity is a type of behaviour that seems complex to us. This reflects the fact that complexity theory does not have the capacity of fully explaining the complexity of the surrounding world yet. Many people interested in these issues (including the author of this work) think that complex systems possess a number of common properties, which can be studied irrespective of the physical nature of the system. Accurately identifying these properties and determining necessary and sufficient conditions when they may occur are the major

distinguished "known unknowns" and "unknown unknowns". challenges that complexity theory has to face. Future successes in overcoming these challenges will inevitably narrow the definition of complexity. The projected benefits for development of science and technology stemming from understanding complexity are very substantial.

\subsection{Technological Evolution}

Common interpretations of innovation and technological development can be divided into three major categories

- Routine: technological progress is accumulated as the result of systematic effort and step by step research to improve product performance

- Heroic: technological progress is achieved by prominent inventors people that possess unique qualities and can overcome initial scepticism of the inertial society

- Evolutionary: inventions are interpreted as "mutations" of our knowledge that are then subject to selection process resulting in either acceptance or rejection of the inventions by the society

While the routine approach is a viable model for incremental improvements, it is unlikely that major technological breakthroughs can be achieved by a large number of engineers merely performing their routine day-to-day tasks. Radical innovations are not always embraced by managers and immediately adopted by the society, hence the role of the inventor in significant innovations is very important. The pattern of inventions often resembles more a complex spiral or an erratic walk through a dark forest rather than a triumphant march along a broad and straight one-way avenue of technological progress.

The term "technological evolution" was introduced by Czech philosopher Radovan Richta (1968) and evolutionary views on technological development have tended to become more common in recent years (Berkun, 2007) replacing the routine and heroic interpretations. Comparison of technological and biological evolutions is given in the Figure 1. The original version of this figure given in Klimenko (2008) has been modified to include purposeful actions of technology developers (i.e. intelligent engineering design) that can replace some 
segments of the evolutionary generation and selection. This replacement is common for achieving relatively minor improvements, while bypassing large evolutionary steps might be increasingly difficult or risky. For example, the same old popular model of a car but with better fuel efficiency will undoubtedly be welcomed by the consumers, while consumer acceptance of an innovative electric vehicle is much less certain. Overall, the evolutionary model better fits technological developments on a grand scale --- these developments are complex and difficult to predict. The intelligent design model is most suitable for smaller incremental improvements that do not affect complex interconnections and can be achieved by a purposeful action of an engineer or a design team.

According to the Weismann barrier, which prohibits inheritance of accrued traits, the intelligent design option is not generally available to biological systems (we do not consider artificial selection and genetic engineering) while different sections of technological evolution can, at least in principle, be assisted or replaced by intelligent action. A professional engineer not only generates an idea but also immediately puts it to the test of his intellect and experience. As the result, the pace of technological evolution is much faster than that of biological evolution. Experimental investigations (Imhof and Schlotterer, 2001) indicate that less than $0.01 \%$ of biological mutations can be beneficial. The number of beneficial inventions (i.e. inventions that are implemented and accepted by the industry and the society) can be roughly estimated by the percentage of commercialised patents, which appears to be around 5\% (Klimenko, 2008). Hence intelligent action and professional experience make technological progress substantially more efficient, yet not fully predictable due to a high degree of complexity of the process

\subsection{Advanced Engineering}

Advanced engineering is a sector of engineering practice that goes beyond the domain of basic engineering, which is constructed around conventional or routine application of already known engineering principles. Advanced engineering encompasses engineering research, technology strategy, systems engineering, industrial operations research and any other engineering activity that requires ingenuity, inventiveness, leadership, interdisciplinary knowledge of fundamentals and advanced abilities. Existence of links between complexity of industrial development and the science of complexity has been commonly recognised (Hall, 1962; Heylighen et. al, 1999) and is discussed further in this section.

Engineering Research. Technological progress follows major scientific discoveries but also involves a substantial volume of engineering research. Success in research cannot be guaranteed by routine application of known procedures and algorithms -- an element of uncertainty is present in any attempt to discover new knowledge. Technical competency in basic engineering allows us to obtain predictable positive results, while engineering research, which requires innovation and is not fully predictable, belongs to the domain of advanced engineering. Innovative companies have to be involved in both basic engineering, which is aimed at applying and maintaining the existing technological level, and in engineering research, which is needed to attain technological progress. Engineering research can be distinguished from research in science by greater emphasis on practical goals in engineering, which is typically combined with using a broad spectrum of research tools not confined to a particular scientific discipline.

Engineering research typically covers fields that are understood very well from the perspective of the relevant fundamental science, but where complexity prevents us from simple conversion of this understanding into specific predictions. For example, we have a good understanding of the fundamental principles of fluid mechanics, thermodynamics and kinetics of common chemical reactions, but this does not guarantee that performance of a specific combustor can be accurately predicted on the basis of this knowledge. Turbulent combustion is a complex process that involves interactions of transport mixing and reaction processes at different scales. The detailed governing equations that are formulated by fundamental science for reacting flows are known but remain computationally intractable. Due to its practical significance, turbulent combustion is thus one of the areas of active engineering 
research, which is aimed at formulating practical and computationally affordable models as well as giving specific predictions for the performance of industrial combustors.

This situation is quite common: fundamental science determines the principle working of the system elements, leaving the rest to engineering. Simulating properties of complex systems is a challenging problem, even if the behaviour of each system element is fully predictable. It is often engineering research that has to face and practically solve the problem of complexity in technological applications. While the short-time influence of research in science and engineering on industrial development might be limited, its long-term impact on technology is profound.

Technology strategy. For any company engaged in production of technologically advanced products, monitoring the newest technological developments is essential. These emerging technologies and ideas may not immediately be of obvious relevance to current operations of the company but in the long run may bring large changes into the area. Typically, revolutionary changes do not happen immediately after a new technology is invented but, as discussed in Section 3, occur with significant time delays.

Strategic planning of technology development and use is usually performed by relatively small groups of experienced engineers reporting directly to senior management. Their roles involve monitoring company performance against its competitors and identifying potential problems and possible improvements in future operations. Strategic reviews are often conducted with participation of external bodies to ensure greater impartiality of the process.

Technology strategy has to be analysed and formulated in conditions of limited information and significant uncertainty, which is commonly associated with behaviour of complex systems. In these conditions, experience, erudition, high professionalism and original thinking become major tools of analysis. Complexities of technological evolution make correctness of engineering decisions perspective-dependent. Therefore, decisions pursuing any immediate benefits have to be supplemented and balanced by strategic considerations.
Snowden's Cynefin framework emphasises that in complex situations, decisions have to be made under conditions of uncertainty, where knowledge is fragmented and incomplete (Snowden et al 2007). This framework distinguishes four types of such conditions (often called four spaces or four realms) ranging from simple to complex:

- known -- the realm of solid scientific knowledge

- knowable -- the realm of science where additional information, inquiry or research are needed

- complex -- knowledge is at best qualitative, each situation has features that are unique and unpredictable

- chaotic -- the realm of completely unpredictable events lying beyond the domain of available experience

Cynefin suggests different strategies of decision making for different conditions (French, 2013). For example, extremely complicated chaotic space can render any coherent strategy impossible due to inherent unpredictability of the outcomes in these conditions. Cynefin advises to act first, then sense and correct further steps. Note that "chaotic" (i.e. unknown, unpredictable and uncertain) is not synonymous with "random" (i.e. governed by simple probability laws and not by anything else) in this context.

Systems Engineering (SE) is an interdisciplinary approach within the engineering discipline that enables the realisation of successful systems in the context of modern technological developments and operations. The term "systems engineering" was probably invented by Bell Labs during the demanding times of the Manhattan project (Dommasch and Laudeman, 1962). In his seminal work that introduced the key principles of systems engineering (SE), Arthur Hall (1962), sees SE as an industrial version of operations research, which is linked to evolutionary complexity, management and science. This book impresses the modern reader by the depth of its analysis as much as it did 50 years ago. In early publications of the 1950 s and 1960s, SE is seen as a broad spectrum of methods that enable efficient control over advanced engineering projects, which can be characterized as large, multidisciplinary, challenging and innovative. 
The following decades brought a more specific and more practical interpretation of SE emphasising technical leadership and systems management (NASA, 1995) as well as its world-wide industrial use (INCOSE, 2013).

SE can be interpreted as an engineering discipline, which is aimed at exercising intelligent control over evolutionary complexity in industrial environments. Whenever possible, it replaces less effective technological modification/selection process by more effective intelligent design. While complexity theory is aimed at understanding complexity, the goal of $\mathrm{SE}$ is managing complexity. As illustrated in Figure 2, complexity theory sees a system controller as part of the system that he (or she) manages while SE is formulated from the perspective of this systems controller. The distinctive feature of SE is that it is aimed more than the other areas of advanced engineering at exercising an effective organisational and technological control over complex developments. A practical approach thus prefers to remove, as much as possible, all sources of uncertainty from SE, which should guarantee that the formulated goal is achieved before the preset deadline. The science of complexity is, on the contrary, is very much concerned with the fact that uncertainties are inherently present in complex systems (French, 2013). Stuart et. al. (2013) have suggested a quantitative approach to decision-making that incorporates uncertainty by considering different scenarios while decision-making is conducted within each scenario.

It should be noted that the task of controlling complex environments is not exclusive to SE: economists, directors, and politicians may have to deal with systems that are extremely complex. SE, however, has a clearly defined engineering goal or target. Formulation of this goal generally lies outside the bounds of the discipline: systems engineers do not decide to fly a mission to Mars but only implement this decision, once it is taken at a higher level. The existence of a defined engineering goal does not make SE strategies inflexible or predetermined - complex projects can involve significant research elements, whose results can not be fully predicted. Both technical complexity and organisational complexity are within the SE domain: an industrial system, which performs complex design and production, should also be complex.

Thus from the perspective of complexity studies, SE is a practical engineering discipline, which

- pursues intelligent control of the evolutionary complexities in the industrial environment associated with modern technological development and

- has a clearly formulated engineering goal

\section{Complex Competitive Systems (CCS)}

\subsection{CCS as a field of complexity science}

While complexity studies is a very large and methodologically diverse area, our attention in this section is restricted to a specific type of complex systems called competitive systems. The theory of complex competitive systems (CCS) is a relatively new area, which studies general principles of evolution in systems with competition and has been recently reviewed by Klimenko (2013). In some respects CCS have similarities with complex adaptive systems (CAS -- see Holland, 2006), in other respects CCS and CAS are different. CAS have two major components: competition, which is conducted according to certain preset rules, and random disturbances, which mostly have negative effect on the elements and are called mutations --- the term borrowed from biology.

In the context of modern industrial production, competition mainly refers to the competition of ideas and technical solutions and not to a rivalry between individuals. An existing solution, design or device is compared with its possible replacement and one of them appears to be a winner and the other one is a loser. The winning design makes it to the final product, although the production process involves many rounds of attempted innovations, alterations and improvements at different stages of the process. While formulating a strategy in industrial environment requires a significant amount of practical experience, the decision-makers' familiarity with pitfalls, which are commonly encountered in control of complex systems, is likely to be beneficial for quality of their 
decisions. In this section we review some general properties of CCS that can be relevant to the issues that strategic planners and systems engineers have to deal with, although we need to mention that CCS is a descriptive (and not prescriptive) framework, which is aimed at explaining the functioning of complex systems with competition.

\subsection{Intransitivity and its implications}

The study of competitive systems distinguishes transitive and intransitive advantages. Advantage of $B$ over $A$, which we denote $A \prec B$, is transitive if for any $C$ :

$$
(A \prec B) \&(B \prec C) \Rightarrow(A \prec C)
$$

Alternatively, the advantage of $B$ over $A$ is considered intransitive provided there exists a finite $(n<\infty)$ set of $C_{1}, \ldots, C_{n}$ so that

$C_{n} \prec A \prec B \prec C_{1} \prec \ldots \prec C_{n}$

Transitive advantages specified by equation (1) can be seen as absolute: subject to the conditions of the Debreu (1954) theorem, advantage of $B$ over $A$ can be expressed in terms of numerical ranking function $r(A)$

$$
(A \prec B) \Leftrightarrow(r(A)<r(B))
$$

which specifies that that $B$ has higher rank than A. Obviously, ranking is impossible for intransitive advantages since (2) requires that

$r\left(C_{n}\right)<r(A)<r(B)<\left(C_{1}\right)<\ldots<r\left(C_{n}\right)$

which is impossible in conventional mathematics. In economics, the ranking function is customarily called "utility". Situations implying intransitive choice may seem paradoxical and, in fact have been known for long time in the form of the Condorcet paradox (Condorcet, 1785). Intransitivity is also known to pose a problem of choice in democratic elections (Arrow, 1951). Intransitive choices are common or even ubiquitous in nature (especially when there exist multiple selection criteria) and can be identified as causes of complexity in competitive systems (Klimenko, 2012, 2013).

In globally intransitive systems, one may distinguish another useful property called current transitivity (Klimenko, 2013). Current transitivity refers to the cases when the current state of a system is restricted to subdomains complying with property (1) although the overall domain of the system evolution is intransitive. Current transitivity produces impression of transitive compliance, when considered from a short-term perspective, but is essentially intransitive in a long run. The example shown in Figure 3 is transitive within the current distribution and behaves locally as a transitive competitive system moving slowly towards higher competitiveness. Gradual increase of competitiveness, which is common in transitive competitions with infrequently positive mutations, is called competitive escalation. The competition in this example is globally intransitive and its evolution is cyclic --- the distribution of elements eventually arrives to point A that the system has started from. While the cycles shown in Figure 3 are linked to the cyclic geometry of the domain, another example of cyclic behaviour in a fully connected domain is considered in Section 3.4.

In the context of an industrial environment, transitive decisions increase product ranking and guarantee unconditional improvements; they do not need to be reconsidered in the future under normal circumstances. For example, modification of an engine design with the sole result of improving car fuel efficiency is a transitive improvement. While engine modification improving fuel efficiency but compromising performance of the engine is intransitive. In simple terms, a transitive improvement always represent a positive change, which always remains positive even when considered from any other perspective.

The complexity of modern technology, however, makes black and white assessment of alterations and innovations quite difficult. Development decisions involve evaluation of many competing factors as well as balancing different or even contradictory requirements. Practically, this means that decisions taken in complex conditions of modern industrial production have a good chance of being intransitive. The intransitive decisions fit a particular comparison of different designs undertaken at a given moment of technological development but do not necessarily mean that the options judged as inferior at present can be completely discarded from consideration in the future.

Many intransitive decisions can be easily found in the fashion industry. Each new 
costume design appears to look better or to be more convenient than the previous one, yet fairly similar fashions tend to reappear within a decade or two. The fashion industry is, of course, a rather extreme example of flourishing intransitivity (note that even this industry does have a degree of transitive development: people now dress differently than, say, a hundred years ago). Intransitivities, however, are not restricted to fashion and can be found in hard-core engineering industries. For example, premixed combustion and diesel cars were for long time considered as impractical or inferior options. Yet now the best low NOx emission combustors are premixed or partially premixed and around $50 \%$ of cars sold in the European market have diesel engines. Practically, technological evolution may involve a monotonic progress in one (transitive) direction and at the same time a repeating cycle in the other (intransitive) direction (see figures in Klimenko, 2013).

The theory of CCS explicitly links complexity and intransitivity - a good and well thought out decision taken in a complex industrial environment has a good chance of being intransitive. Practically, this aspect of decision making is often neglected and there is a common perception that if option $A$ has been considered, analysed and judged as inferior, there is no need to consider this option ever again. When occasionally this proves to be wrong, it is common to infer that the first decision was a mistake (hence, people involved in making the original decisions would typically object to re-examining previously discarded options). Intransitivity, however, makes it possible that the first decision was well-thought and absolutely correct and yet option $A$ needs to be considered again when circumstances change. Correctness of a decision is not the same as its transitivity. Decision-makers need to be aware about intransitive aspects of complexity and modern industrial environment is complex. It always pays off to think not only about the present but also about the future. While making an intransitive decision, it is a good practice to note that although a particular option is not suitable now, it should nevertheless be kept in mind for future considerations.

\subsection{Generalisation for competition events with multiple elements}

In the previous subsection, we consider pairwise competition between multiple particles. This means that elements form interacting (competing) pairs and for each pair the winner and the loser are determined. For example, $A \prec B$ indicates that $B$ is the winner in competition with $A$ and the interaction group of $A$ and $B$ is ordered as $(B A)$.

In some applications (and decision-making is one of these), it might be useful to consider interaction groups of more than two elements. If competition is transitive, the binary (pairwise) comparisons are easily generalised for groups of arbitrary size by deploying absolute ranking of the elements. For example the binary relations $A \prec B \prec C \succ A$ imply that the possible pairs are ordered as $(B A),(C B)$ and $(C A)$. Since these relations are transitive, it is immediately obvious that the interaction group of three should be consistently ordered as (CBA). Intransitive competition, on the other hand, does not offer any obvious treatment of multi-particle groups. Indeed, the binary ordering $(B A),(C B)$ and $(A C)$, which corresponds to intransitive competition rules $A \prec B \prec C \prec A$, does not give an indication of how the triplet of $A, B$ and $C$ should be ordered. We can, of course simply define an ordering for every possible group, but this seems to be excessively flexible, quite arbitrary and inconsistent with the transitive case.

Let real-valued function $R(A, B)$ be the equivalent co-ranking of the element preferences, that is

$$
R(A, B)<0 \quad \Leftrightarrow \quad A \prec B
$$

for any elements $A$ and $B$. By definition, the element co-ranking function is antisymmetric $R(A, B)=-R(B, A) \quad$ and $\quad R(A, A)=0$. Consider group $G$ comprised of $k$ elements $C_{1}, \ldots, C_{k}$. The relative ranking of element $A$ with respect to group $G$ is given by

$r_{G}(A)=\frac{1}{k} \sum_{C_{j} \in G}^{k} R\left(A, C_{j}\right)$

In this definition, element $A$ may also belong to group $G$. In a multi-element competition event, Let $G$ be a competing group. In this 
group the elements are to be ordered according to their rankings relative to the same group, that is

$$
\begin{aligned}
& \underbrace{\left(C_{a} C_{b} C_{c} \ldots\right)}_{G} \Leftrightarrow \\
& r_{G}\left(C_{a}\right) \geq r_{G}\left(C_{b}\right) \geq r_{G}\left(C_{c}\right) \geq \ldots
\end{aligned}
$$

In the case of pairwise competition, ordering (7) is the same as the previously defined ordering (5). Equation (7) is also consistent with multi-element transitive competition. In transitive competition the co-ranking function is expressed by equation

$$
R(A, B)=r(A)-r(B)
$$

in terms of absolute rankings of the arguments.

\subsection{Technological cycles}

Complex competitive systems (CCS) tend to evolve in cycles. Existence of technological, economic and historic cycles is well documented in the literature (as reviewed by Klimenko, 2008, 2010, 2012). Technological waves can occur at different time scales. At the largest time scale of around 50 years, five Kondratiev waves are distinguished (Kondratiev, 1925). Carlotta Perez (2010) have understood and interpreted these waves not as mere oscillations but as technological surges of industrial activity associated with emergence, maturity and decline of new industrial sectors. There exist technological cycles of different durations and magnitudes, with the product or project lifecycles (see NASA, 1995) being one of the shortest. The generic form of cycles of emergence, growth, maturity and decline in systems involving competition is called the leaping cycle. A typical technological cycle is schematically depicted in Figure 4.

According to the theory of CCS, the main cause of these cycles is hidden in intransitivity of competitions: the prevailing structure has to be strong against existing competitors while its overall structural resilience and resistance to potential competitors (i.e. competitors not currently present) may decline due to intransitivity of the competition rules. This process, where loss of competitiveness is often hidden beyond apparent strength of the dominant structure, is called competitive degradation. When this loss becomes critical, a small disturbance can cause dramatic changes in the system, such as falling into the basic ground state or, in more complex cases, collapse of the dominant structure. Then a new structure has a chance to grow in the vacated space until it reaches a dominant position. The cycle repeats itself. Computer simulations of a complex system with 64 thousand elements, which are engaged in intransitive competition localised in physical space, have been shown to display repeating leaping cycle (Klimenko, 2013, electronic supplement with figures, explanations and a video).

These abstract considerations have a direct relevance to technological evolution. Typically a new technology does not immediately take over an incumbent technology but has to survive with a relatively small share of the market. Under these conditions the incumbent technology may keep its dominance for a long period of time up until it finally collapses. For example, diesel locomotives appeared in the 1920 s but only in the 1950 s steam was promptly and en masse replaced by diesel in American railroads and then worldwide (see, for example, Klimenko 2008). It is often the case that nothing obvious indicates imminent collapse of aging dominant technology, which can be absolutely unexpected. The industry of the 1950s, which produced excellent steam locomotives for many decades, was not able to adapt to rapid changes and went into receivership. It is needless to say that decision-makers should be aware about potential pitfalls of technological evolution.

There are different aspects of competitive degradation that may weaken previously successful technologies and companies. Some of them, bureaucratisation for example, can be identified and tackled. A bureaucratised company or organisation follows all formal rules and performs quite well on paper but in fact stalls initiative, terminates innovation and practices "business as usual" as the best possible policy. The practical remedy can be found in reorganising the system by adapting it to the current environment and deploying more horizontal and project-oriented structures (although the effect of numerous, excessive reorganisations can be negative).

While certain aspects of competitive degradation (such as bureaucratisation) can and should be tackled, believing that competitive degradation can be eliminated completely would be a mistake. Some of the 
degradations are inherent and can not be separated from the correct functioning of the system. Let us consider example of the degradation mechanisms for technological applications. Any technology initially has plenty of space for improvements. As technology reaches maturity, any further improvements become more and more difficult. Competition between companies, however, pushes for further improvements, which can be achieved by reducing some of the margins. Each of these margin reductions improves performance and is a very reasonable measure, which, if performed through a proper engineering process, does not affect stability of operation. As time goes by, many of these changes accumulate and make operations quite sensitive to the operating conditions. A change in environment, which may not be that dramatic on its own, may cause malfunctions if margins are small. For example, a modern efficient gas turbine would be much more sensitive to quality of its fuel than similar turbine designed a few decades ago.

This demonstrates the treacherous nature of competitive complexity: in order to stay at the same place (i.e. maintain the same market share), a company must run fast (i.e. innovate and develop) but these improvements can push the existing technology into the corner of being too costly and difficult to handle. If a new technology appears, collapse of the old technology may become inevitable. Hence companies can not stop or they would lose their profits to their competitors but, if they keep moving, the industry may evolve towards the difficult times of abrupt technological changes.

While technological shifts may be disastrous for some of the companies, these changes are important parts of the technological progress and their effect should be positive in the long run. Can the cyclic nature of technological evolution be completely eradicated? Probably not, if we do not wish to terminate the process of technological development, but certain lessons can always be learned from the past and some practical remedies can undoubtedly be suggested. A company should not assume that its current technology will continue forever and should have a technological backup plan. Strategic planning and monitoring prospective and emerging technologies is most essential. This does not mean that anything that might have good prospects in the future should be immediately implemented (recall that advantages of a new technology are likely to be intransitive). Premature embracement of an innovation can be very costly, while failure to notice and react to approaching changes might become deadly. Large companies are aware of this and often buy and store emerging ideas indiscriminately, just not to miss the train departing with a new generation of technologies, but often still miss it. It is well-known example that Steve Wozniak --- one of Apple's founders --- came up with his design of a personal computer to his former bosses at HP several times and was turned away (see, for example, Berkun, 2007).

The world of intransitive competition, which is studied in CCS, does not have the absolute right and the absolute wrong. A measure that improves the short-term competitiveness of a company may be a disaster in the long run and vice versa. Combining tactical considerations with strategic analysis is essential for long-term survival.

\subsection{CCS modelling of the growth vs. risk dilemma}

The problem of balancing economic growth and economic risk has been repeatedly discussed in economic literature (Ladwig, K., 2001; Hallegatte, S., 2012). From the perspective of CCS, the contending requirements of keeping the growth high and the risk low can induce intransitivity, which ultimately results in cyclic behaviour.

The values proportional to growth and risk are denoted here by $Y$ and $X$ correspondingly. Note that $Y$ can be interpreted as production rate or market expansion, while $X$ can refer to losses, or costs, or other similar quantities. The gray area in Figure 5 shows the boundaries of the allowable domain, indicating that a stronger growth is associated with a higher risk, which increases faster than the growth. This risk is quite small between points $\mathrm{A}$ and B but becomes very substantial towards point C. The allowable domain is specified by the minimal possible risk

$$
X \geq Y^{n}, \quad n=3
$$

indicating the minimal possible risk $X$ associated with a given growth $Y$. The 
competition rules are specified by the following equivalent co-ranking function

$$
R(A, B)=\left(Y_{A}-Y_{B}\right)-\left(X_{A}-X_{B}\right)^{k},
$$

where $k=3$. While competition with $k=1$ would comply with (8) and thus be transitive, equation (10) with $k=3$ specifies competition rules that are densely intransitive (i.e. intransitive triplets can be found in vicinity of every point). The exponent $k=3$ in (10) indicates that a small risk is considered as a reasonable price paid for a growth, while any large risk becomes a major concern that overweights growth. Two categories of random mutations are deployed in the simulations: frequent small and infrequent large. Mutations are not allowed to violate (9). We stress that all of the problem parameters (these are the constants characterising competition and mutations) do not change during the simulations, while $X$ and $Y$ affect competition only as specified by equations (9) and (10). In fact, $X$ and $Y$ are generic and can represent any other quantities (not necessarily economic) that possess similar properties.

The solid lines in Figure 5 illustrate trajectories of mean $X$ and $Y$, while the dashed lines show the shape of the cycle expected from qualitative considerations. In the context of computer simulations, competing elements can conventionally be called particles (which are very similar to notional particles used in modelling of turbulent reacting flows - see Pope, 1985). The red dots show the distribution of particles at a selected moment -- 10000 particles are used in the simulations. The state A gives plenty of opportunities for competitors to expand their businesses with minimal associated risk. During these initial stages risk is not the main point of concern and a few trajectories do not follow the minimal risk path. The current distribution of competitors escalates from A to B into the area of further aggressive expansion.

If this competition was transitive, the expansion would continue forever and the most aggressive competitors would always be the winners. Infinite growth, however, is impossible as expansions are constrained by many factors (including the laws of physics), and this is reflected by increasing risk. What might seem initially as pure competitive escalation of healthy growth is in fact accompanied by the stealthy competitive degradation represented by the increasing risk. As the distribution moves from point $\mathrm{B}$ to point $\mathrm{C}$, the risk keeps rising, making defensive strategy A more and more attractive. The situation becomes unstable, as any defection from expansionist to defensive strategy causes an avalanche of followers, quickly bringing the system into its ground state A. In this cycle, we might know from the start that the original growth is in fact intransitive; it will not last forever and is destined to collapse. This knowledge, however, does not exempt the competitors from following the current trend and pursuing expansion as, otherwise, they may become losers straight away, without even reaching point B.

\section{Industry Competition: Example and Discussion}

\subsection{Detecting intransitivity: competition in automotive industry.}

In this example, we will try to detect intransitivity in a real-world industrial competition. The US car market has the sales data over more than 50 years available in public domain (WardsAuto, 2013), which makes it a suitable subject for our analysis. Economic analysis of the market is, of course, not intended here and can be found elsewhere (see Berry et al, 1995).

Our major difficulty is that we do not know the number of buyers switching from one particular car manufacturer to another, but only the total sales. We nevertheless might be able to detect intransitivity due to the existence of time delays between company decisions and the resulting market movements. Another difficulty is existence of the obvious long-term trends in the US car market, which are reflected by the increase of the market share held by Japanese manufacturers, as well as the presence of random short-term oscillations. We are interested in statistically stationary variations, which nevertheless represent reliable periodic trends. For this purpose the data are de-trended and smoothed by highorder polynomials. Standard Matlab tools are used for this purpose. Figure 6 represents the sales data for the six largest car manufacturers in the US car market. The solid lines indicate 
smoothed approximations and the dots show the linear trends removed.

Table 1 presents the correlation coefficients between the market shares for all six companies. The original data are used above the diagonal, while correlation coefficients evaluated for the de-trended data are located below the diagonal. The 3 groups can be distinguished among these manufacturers on this basis: Japanese manufacturers, Ford and Chrysler, while the largest manufacturer, GM (General Motors), forms a group on its own. Among Japanese manufacturers, Nissan has abnormally high detrended correlation with GM and abnormally low de-trended correlation with Honda.

\begin{tabular}{|l|c|c|r|r|r|r|}
\hline & GM & Ford & Chrysler & Toyota & Nissan & Honda \\
\hline GM & 1.00 & 0.74 & 0.10 & -0.94 & -0.81 & -0.95 \\
\hline Ford & -0.14 & 1.00 & 0.51 & -0.89 & -0.83 & -0.83 \\
\hline Chrysler & -0.15 & 0.67 & 1.00 & -0.30 & -0.41 & -0.26 \\
\hline Toyota & -0.13 & -0.78 & -0.63 & 1.00 & 0.90 & 0.98 \\
\hline Nissan & 0.39 & -0.45 & -0.64 & 0.35 & 1.00 & 0.88 \\
\hline Honda & -0.17 & -0.47 & -0.57 & 0.67 & 0.10 & 1.00 \\
\hline
\end{tabular}

Table 1. Correlation coefficients for market share historical records for the six largest manufacturers in the US car market.

While we test groups of three for intransitivity, we need to exclude joint increases and decreases of the market share by normalising each manufacturer by the total share of the group. By doing this, we map the three-dimensional states of the group on the two dimensional domains enclosed by the triangles shown in Figure 7. The competitions within two groups demonstrate intransitivity. The cyclic behaviour Toyota $\rightarrow$ Nissan $\rightarrow$ Honda $\rightarrow$ Toyota is most prominent among all manufacturers but a similar cycle GM $\rightarrow$ Ford $\rightarrow$ Chrysler $\rightarrow$ GM can also be detected. It seems that the latter cycle is stretched horizontally and moderated by the fact that $\mathrm{GM}$, as the largest manufacturer, has to act against both Chrysler and Ford at the same time. While the American "Big Three" take their turn in the cycle strictly according to their market shares, this seems not to be the case with the Japanese "Big Three" since Nissan has a smaller market share compared to Honda. In fact, Nissan used to take the second place among Japanese "Big Three" but was overtaken by Honda in the late 1990s. It is interesting that these companies still behave as if Honda had never overtaken Nissan.

As shown in Figure 7, the competition between GM, Nissan and Chrysler is not cyclic: in most cases GM and Nissan simply benefit from Chrysler's downs but have to give their earnings back when Chrysler recovers. Competition between three groups GM, Ford + Chrysler and the Japanese "Big Three" does not display any consistent pattern. Figure 7 does not indicate existence of a coordinated strategy within these groups; in fact the manufacturing companies seem to be more preoccupied with competition within the groups than between the groups. Bresnahan (1987) suggested that some unusual price changes in the American car market of the 1950 s can be explained by tacit collusion. In the rest of this paper, we do not invoke the collusion hypothesis and suggest explanation for the cycles on the basis of unrestricted albeit intransitive competition.

\section{2. "Big three" and intransitive competition.}

Consider three car manufacturers A, B and $\mathrm{C}$ ordered according to their market share (with A being the largest). From the perspective of the second largest manufacturer, $\mathrm{B}$, the largest market share occupied by A seems to be a very attractive option for growth. It would be logical for B to adjust its market strategy in attempt to attract some fraction of that share. For example, B might increase the size of its major car model to match the size of a very popular car produced by $\mathrm{A}$ and at the same time still offer a better fuel economy. The plan seems to work: Figure 8 illustrates that $\mathrm{A}$ loses some of its customers to B.

The story, however, does not end here. While many old customers of company B, still buy their favourite brand, others feel that the new model is a bit too large for city travel. This opens an opportunity for $\mathrm{C}$ to capture the disaffected customers of $\mathrm{B}$. Company $\mathrm{C}$ launches its new model, that has the smaller size of the old B model and, in addition, offers extra-features at no extra-cost. This seems to work well --- Figure 8 indicates that some customers of B defect to C.

This, however, is also not the end of the story. Company A has recently suffered some shrinkage of its market share and is planning a 
new strategy. This company is going to use it superior market power, economies of scale and financial resources to regain the lost positions. Its new model is going to be sold with many extra features standard, with improved performance and at a reduced price. Company $\mathrm{C}$, which has just made significant investments into its new model, has a difficult time matching this price and loses some of its customers to A. The circle is now completed.

While the particulars of the competition might be different in the real world, the story, depicted in Figure 8, illustrates plausibility of intransitive competition when $\mathrm{B}$ wins from $\mathrm{A}$, $\mathrm{C}$ wins from $\mathrm{B}$ and $\mathrm{A}$ wins from $\mathrm{C}$. There is nothing illogical or suspicious in this situation: each company simply makes decisions, when made, seem to constitute the best possible strategy, and yet the overall system evolves in a cyclical manner.

\subsection{Intransitive decision making}

Intransitive competition can be deemed to be relevant to intransitive strategies pursued by competitors. In this subsection, we continue the example of the previous subsection and consider strategies of company B, which are, perhaps quite simplistically, confined to three options (B1) do nothing, (B2) act against company A and (B3) act against company C. The outcomes of these strategies are illustrated in Figure 9. Option B1 is business as usual and will result in some loss of market share. Option B2 involves upgrading B's popular model to make it more competitive against A's popular model. Option B3 is a price drop combined with outsourcing of some parts aimed at forcing $\mathrm{C}$ to abandon its less profitable models and yield a segment of its market share to B.

The three strategies $\mathrm{B} 1, \mathrm{~B} 2$ and $\mathrm{B} 3$ are assessed according to the three criteria technological progress, market share and budget position -- as shown in Figure 9. Option B2 offers both increase of the market share and technology development and seems to be more attractive than B1. Option B3 comes with a surprise advantage over B2: it offers a larger increase in market share and at a lower cost. Finally it seems that B1 is better than B3: we preserve quality of the manufactured cars and doing this at no extracost. This analysis can be summarised by

$$
B 1 \prec B 2 \prec B 3 \prec B 1
$$

where the symbol of superiority " $\prec$ " indicates achieving advantage in at least two out of three categories shown in Figure 9. We might consider choice between these options as a competition between them and this competition is intransitive.

As we know from the previous subsection, company B has selected strategy B2. This might have happened due to numerous reasons:

- Planning mistake: $\mathrm{B}$ did not foresee the likely response from $\mathrm{C}$ and overevaluated its prospective market share as shown by the open triangle in Figure 9.

- Unpredictable events: $C$ has sold its foreign operation and reinvested its capital into a new domestic model predicting this chain of events was impossible.

- Company values: B knew about the likely response from $\mathrm{C}$ and still selected option B2 after applying the company value system to resolve the decision deadlock expressed by equation (11). (For example, technological progress might be seen as the main prerequisite for future competitiveness of the company and is thus weighted more than the cost and the market share.)

- Strategic considerations: B knows that forcing $\mathrm{C}$ out of business will result in $\mathrm{B}$ facing its largest competitor A alone

- Current transitivity: B knew about the likely response from $\mathrm{C}$ and has selected B2 since strategy B3 was not known or was known but not practically available at the time when the decision was made.

We may conclude that intransitivity is abatable when the future is fully predictable. Indeed, the future states of the available choices can be brought forward in time to the moment of making decisions and subjected to the selection criteria. Intransitivity is then removed by applying a system of values to the present and future states. The situation is more complex when, as in the real world, the future is unpredictable. This unpredictability can be modelled by introducing different scenarios, 
whose probabilities remain unknown and no effective weights can be assigned to each scenario. Stuart et al, (2013) suggested an analytical framework for decision-making under these difficult conditions.

We need to stress that the approach of CCS is descriptive and not prescriptive. We expect that, under the complex conditions of uncertain future, intransitive selections become inevitable. The CCS approach only recognises this possibility but does not imply any recommendation to actually make intransitive choices --- intransitivity may seem illogical to many people. At the same time, understanding the intransitive nature of complex competitions does not necessarily relieve the decision-maker from making intransitive decisions. In the example shown in Figure 5 competition can force competitors to undertake expansionist strategies irrespective of whether they actually wish to do this or not.

\section{Conclusions}

The question of interaction of complexity and industrial production is not a hypothetical question: in the last decades the hi-tech sectors have already faced problems associated with the complexities of modern technological design and development. Irrespective of whether this link between complexity and technological development has been explicitly recognised or not, industries have developed a set of practical a responses to these problems. Systems engineering (SE) is a notable part of this response. At present, the science of complexity is still at the beginning of its development and, it seems, that uncertainty and intransitivity play very significant roles in emergence of complexity. As time goes by, this science will hopefully understand complex systems better and will be able to provide increasingly direct and specific advice.

\section{Acknowledgements}

The development of notional particle methods, which led to conceptualisation of the complex competitive systems framework, has been supported by the Australian Research Council. The author thanks Bruce Littleboy for insightful economic discussions. Constructive comments of the anonymous reviewer are also appreciated by the author.

\section{References}

1. Arrow, K. J..(1951) Social Choice and Individual Values. Yale University Press, USA,

2. Berkun, S. (2007) The Myths of Innovation, O'Reily Media

3. Berry, S; Levinsohn, J. and Pakes (1995) A Automobile Prices in Market Equilibrium, Econometrica, Vol. 63, No. 4, pp. 841-890

4. Bresnahan, T. (1987). "Competition and Collusion in the American Automobile Industry: The 1955 Price War." The Journal of Industrial Economics, Vol. 35, No. 4, 457-482.

5. de Condorcet, N. (1785) Essay on the Application of Analysis to the Probability of Majority Decisions. De L'imprimerie Royale., Paris.

6. Debreu, G. (1954) Presentation of a preference odering by a numerical function. in R. M. Thrall, C. H. Coombs, and R. L. Davis, editors, Decision process, pages 159-165. J.Wiley and Sons,

7. Dommasch, D.A. and Laudeman, C.W. (1962) Principles Underlying Systems Engineering, Pitman Publishing Corporation . New York

8. French ,S. (2013), Cynefin, statistics and decision analysis Journal of the Operational Research Society (2013) 64, 547-561

9. Hall, A. D. (1962) A Methodology for Systems Engineering. Van Nostrand, N.Y.

10. Hallegatte, S. (2012) An Exploration of the Link between Development, Economic Growth, and Natural Risk, The World Bank: Office of the Chief Economist, Policy Research Working Paper 6216.

11. Heylighen, F., Bollen, L. and Riegler, A. (eds) (1999) The Evolution of Complexity, The Violet Book (vol.8) of "Einstein Meets Magritte", Kluwer AP, Boston.

12. Holland, J.H.. (2006) Studying complex adaptive systems. J. of Syst. Sci. and Complexity, 19(1):1, 8

13. Imhof, M. and Schlotterer, C. (2001) Fitness effects of advantageous mutations in evolving Escherichia coli populations, PNAS, vol. 98 no. 3, pp 1113-1117

14. INCOSE (International Council on Systems Engineering) (2013), http://www.incose.org/, accessed in Feb, 2013

15. Klimenko, A. Y. (2008), 'Technological cycles and their impact on science, engineering and engineering education', The International Journal of Technology, Knowledge and Society, 4 (2), 11-18. 
16. Klimenko, A. Y. (2010) Computer simulations of abstract competition. In Proceedings of International Conference on Complexity, Informatics and Cybernetics (IMCIC), vol. 1, pages 97102.

17. Klimenko, A. Y. (2012) Mixing, entropy and competition. Physica Scripta, 85:068201.

18. Klimenko, A. Y. (2013) Complex competitive systems and competitive thermodynamics, Phil. Trans. R. Soc. A, 2013, 371.

19. Kondratiev, N. D. (1925) The Long Wave Cycle (translation from Russian "The Major Economic Cycles"). Richardson and Snyder, NY, 1984.

20. Ladwig, K. (2001) The risk vs. growth balancing act. Collections \& Credit Risk, vol. 6 , Issue 10, p. 58.
21. NASA (National Aeronautics and Space Administration) (1995) , Systems Engineering Handbook, NASA report SP$610 \mathrm{~S}$

22. Perez, C. (2010). Technological revolutions and techno-economic paradigms. Camb.J. Econ., 34(1):185-202.

23. Pope, S. B. (1985) Pdf methods for turbulent reactive flows. Prog. Energy Combust. Sci., vol. 11, 119--192.

24. Snowden D and Boone M (2007). A leader's framework for decision making. Harvard Business Review 85(11): 68-76.

25. Stewart, T.J; French, S. and Rios, J. Integrating multicriteria decision analysis and scenario planning - Review and extension Omega 41 (2013) 679-688

26. WardsAuto (2013) http://wardsauto.com/

\section{Figures}
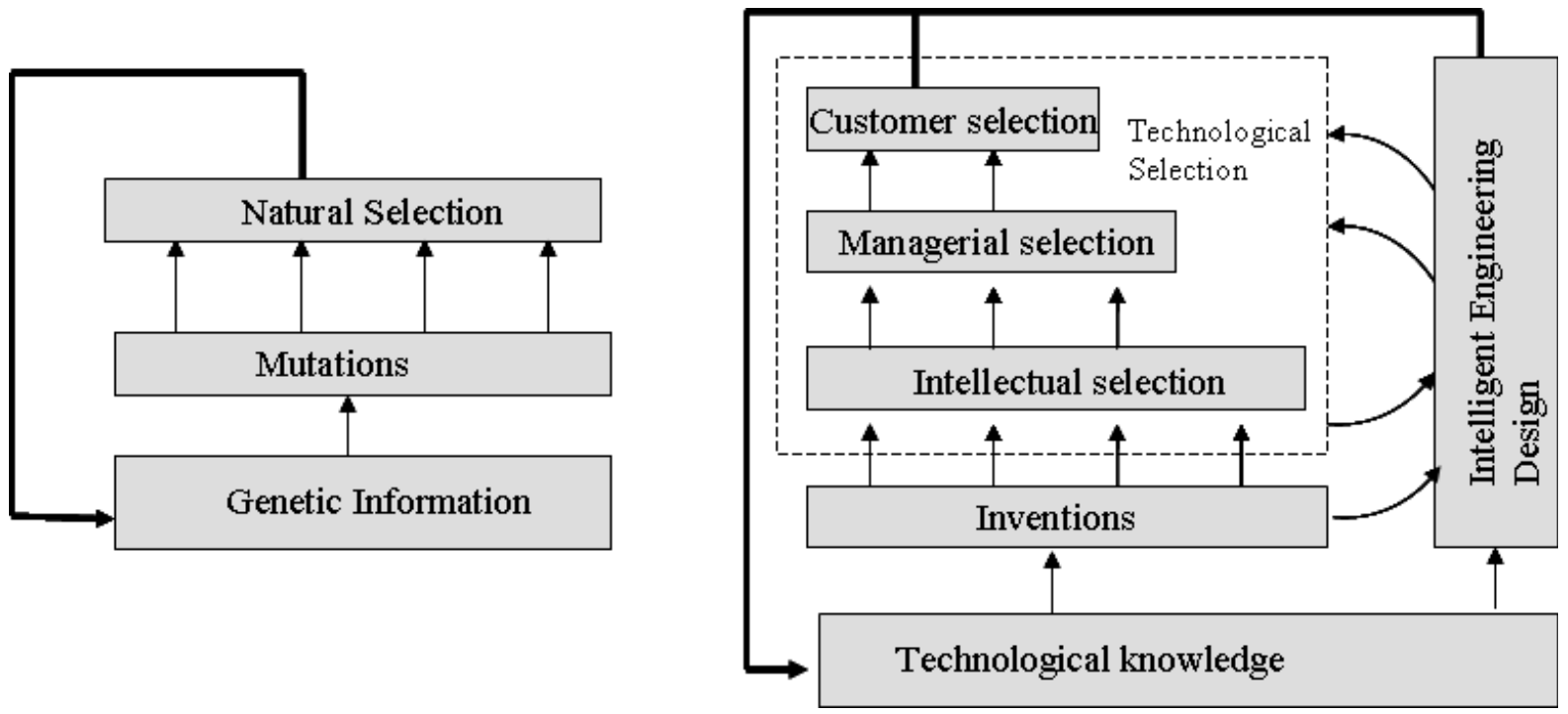

Figure 1. Comparison of biological and technological evolutions. 


\section{Economic conditions, Radical innovations, Strategic planning, Executive decisions}

$\downarrow$

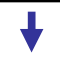

$\downarrow$

$\downarrow$

managing

systems

engineer
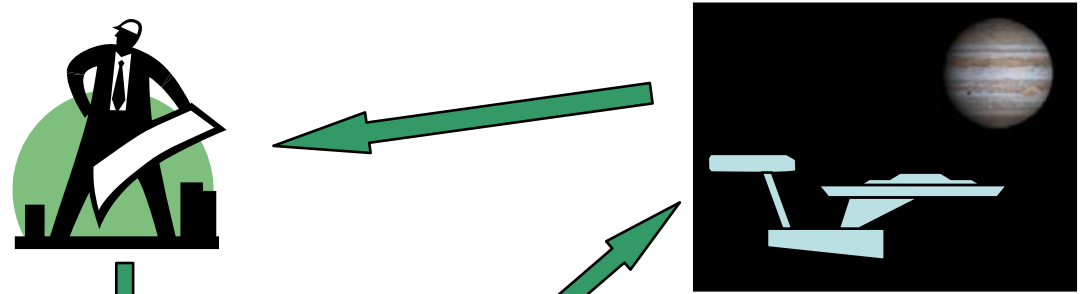

Engineering goal

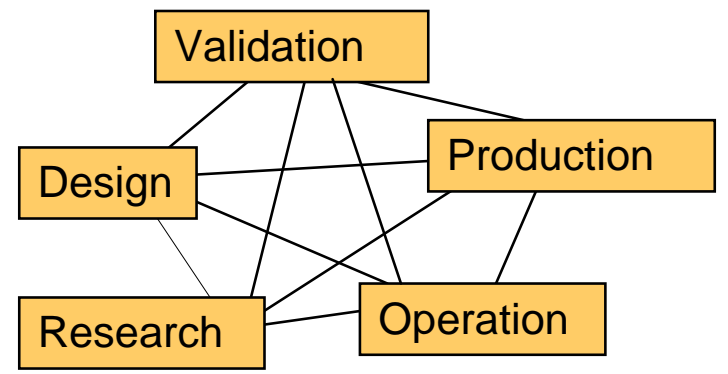

System boundary as

seen by systems

engineers

System boundary from the perspective of complexity theory

Figure 2. Boundaries of systems engineering

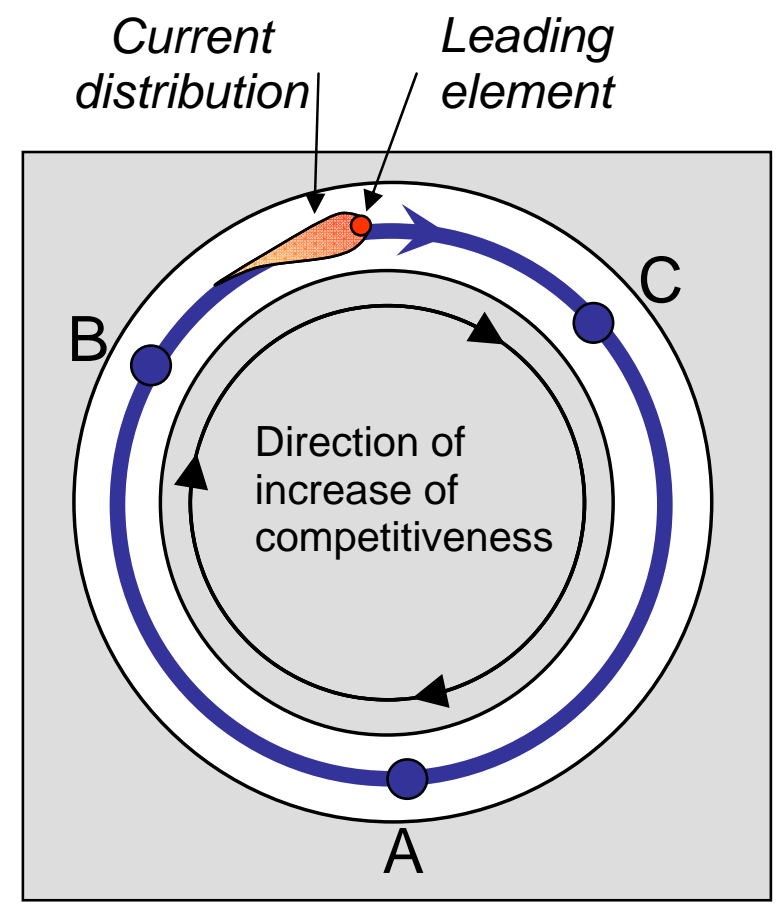

Figure 3. Intransitive competition is a circular domain 


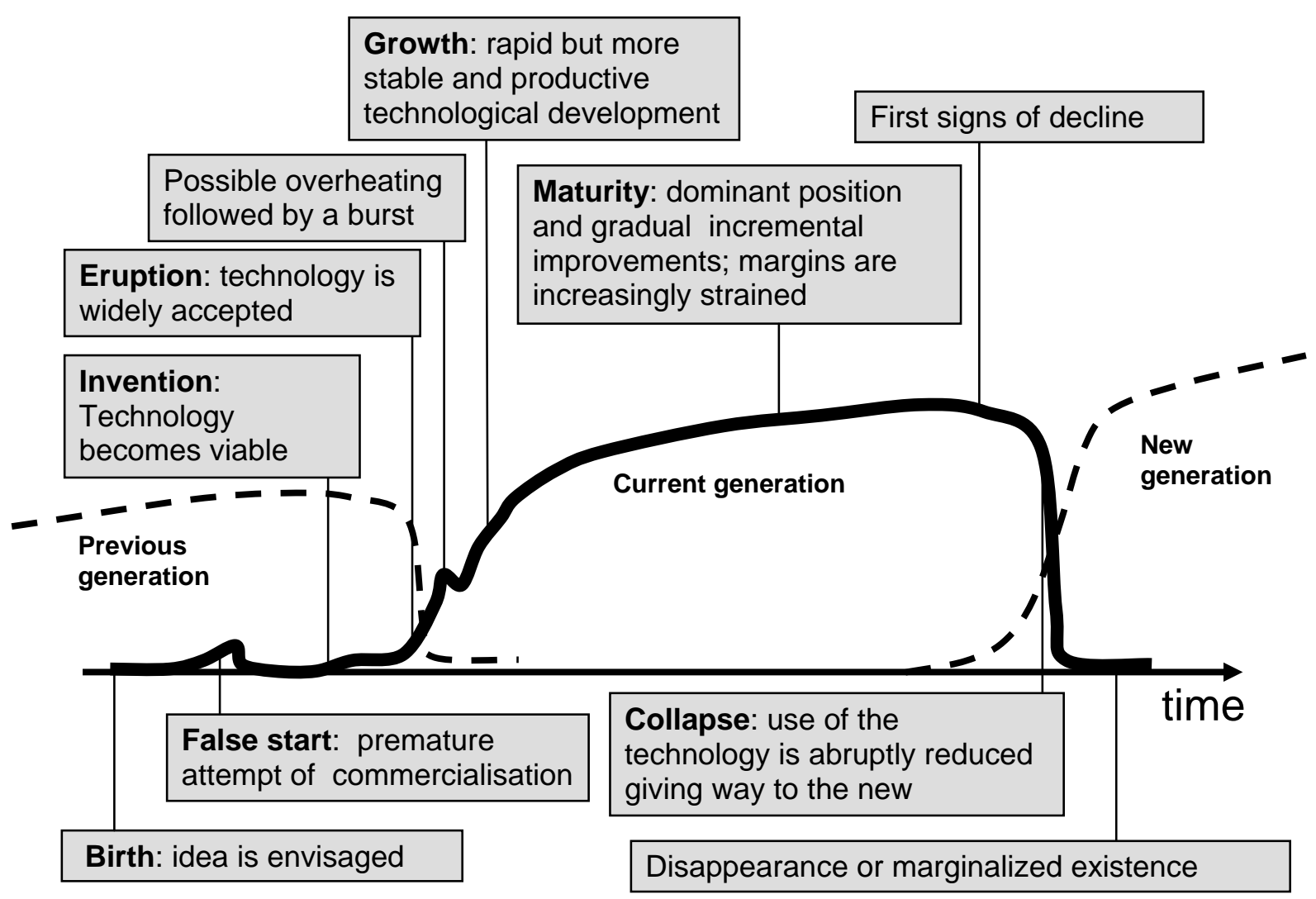

Figure 4. Technological cycles

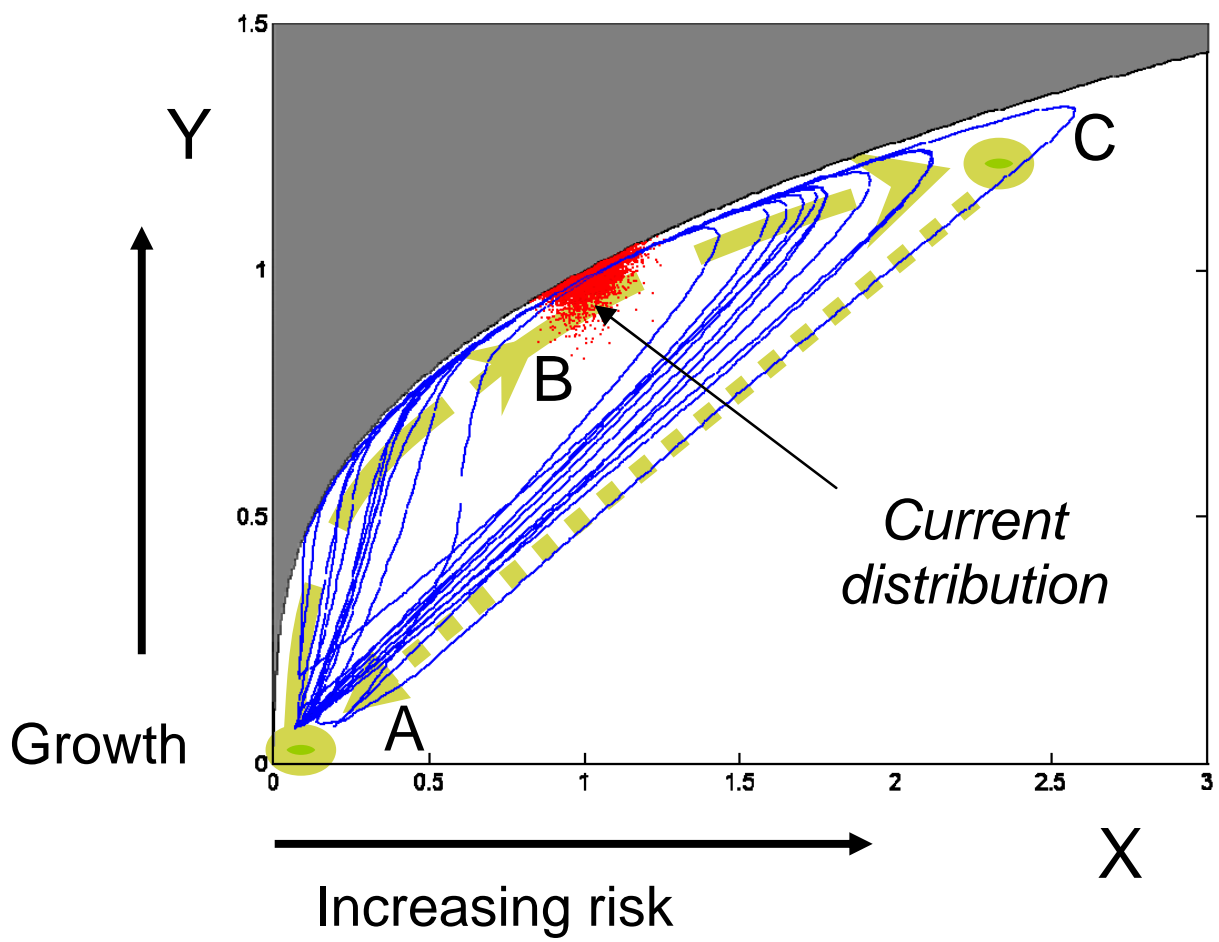

Figure 5. The growth vs. risk cycle 

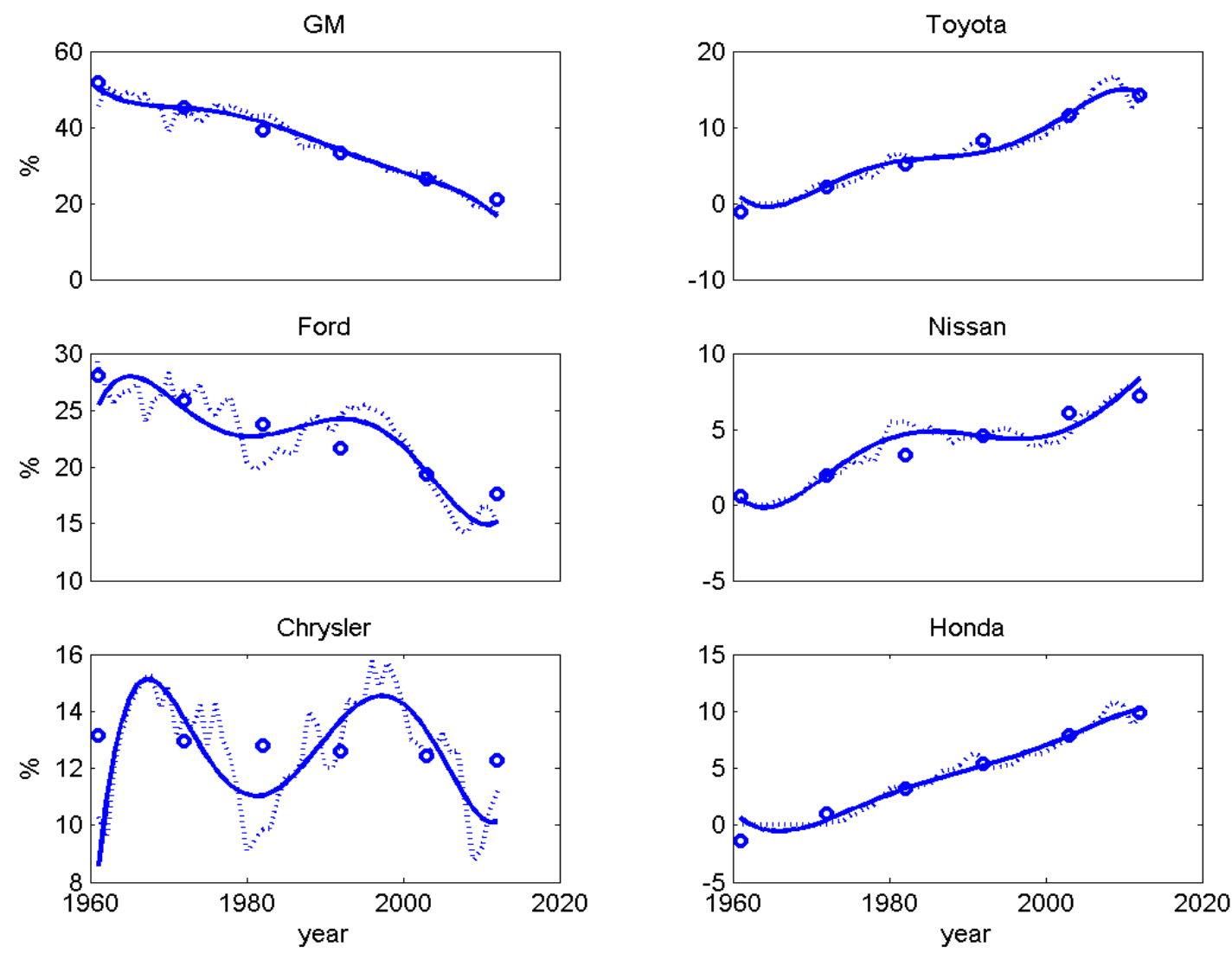

Figure 6. Market share for the six major car manufacturers in the US market
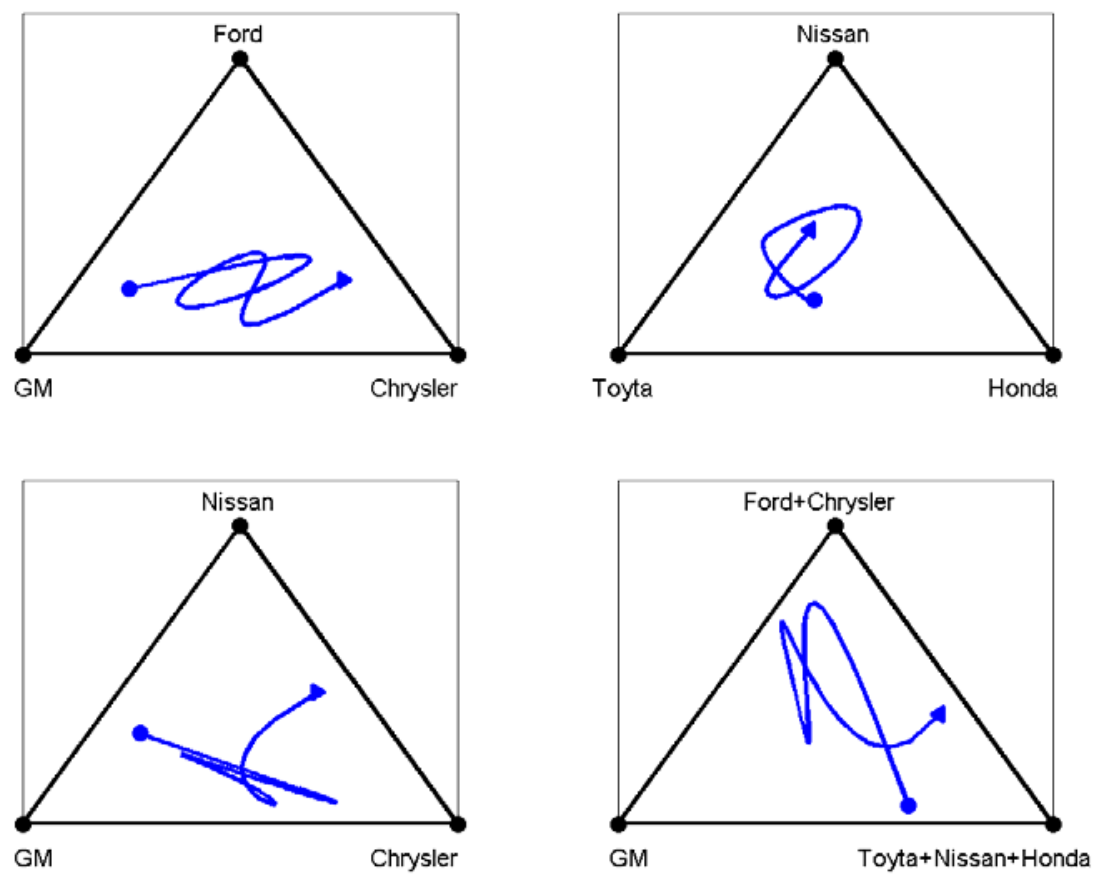

Figure 7. Intransitivity in automotive industry competition. The curves show joint evolutions of the relative market shares for different groups of three companies. Locations closer to a vertex with a company name indicate a higher market share for this company. 


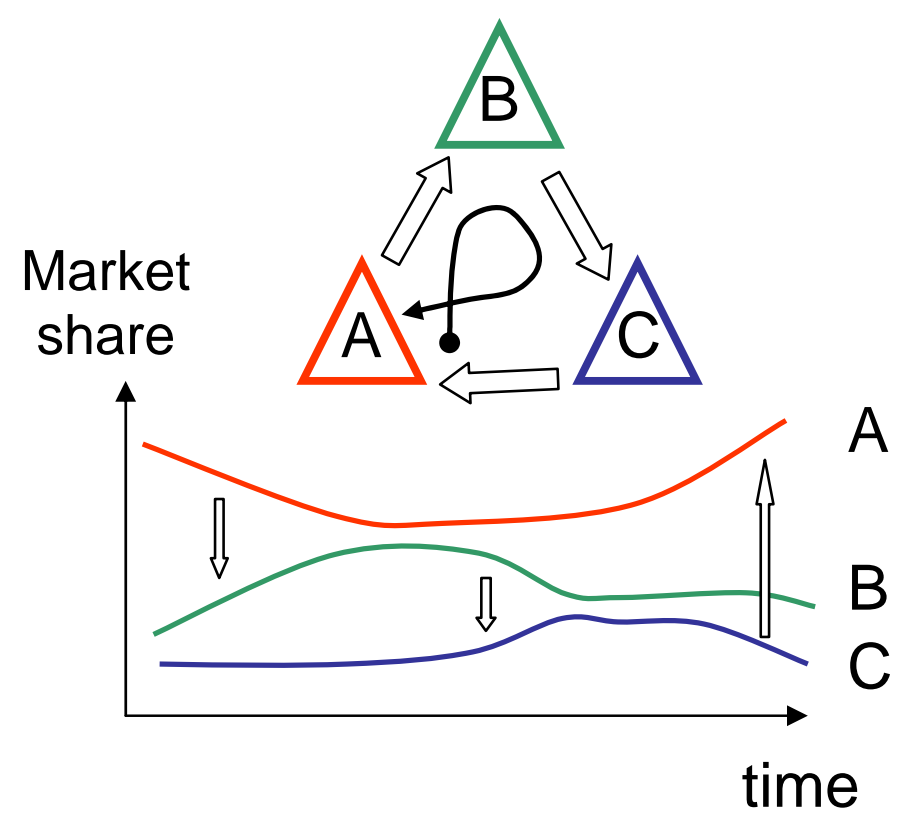

Figure 8. Intransitive competition between "Big three". The bottom figure shows variations of the market share versus time for three competitors A, B and C. The top figure illustrates the qualitative dynamics of the market shares that corresponds to the evolutions shown in the bottom figure.

O B1 Business as usual

$\triangle B 2$ Upgrade the model

$\square$ B3 Drop the price

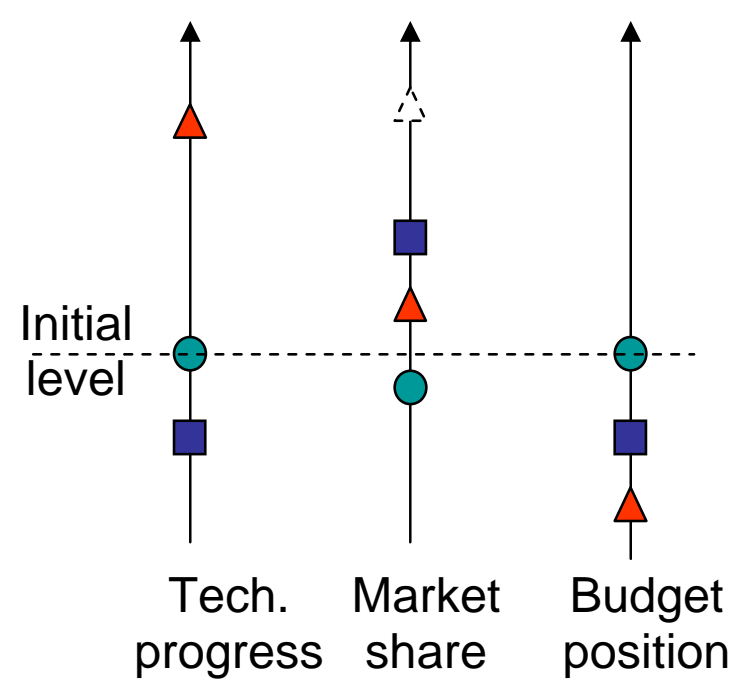

Figure 9. Strategies of manufacturer B and payoffs. The strategies are listed at the top, while the payoff of these strategies with respect to three criteria --- technological progress, market share and budget position --- are shown at the bottom. 